\title{
Úlceras Por Pressão em Neonatos Internados em uma Unidade de Terapia Intensiva Neonatal: Série de Casos*
}

\author{
Pressure Ulcers in Newborns Admitted to the Neonatal Intensive Care Unit: a Case Series \\ Úlceras por Presión en Recién Nacidos Internados en una Unidad de Cuidado \\ Intensivo Neonatal: Serie de Casos
}

Talita Faraj Faria', Ivone Kamada

\begin{abstract}
RESUMO
O objetivo deste estudo foi descrever a ocorrência de úlceras por pressão em recém-nascidos internados em uma Unidade de Terapia Intensiva Neonatal de um hospital público de Brasília, Distrito Federal, Brasil. Trata-se de um estudo exploratório de caráter descritivo, do tipo série de casos, realizado de setembro a dezembro de 2014. Foram avaliados seis recém-nascidos que desenvolveram úlcera por pressão durante seu período de internação na Unidade de Terapia Intensiva Neonatal. Em quatro neonatos, as úlceras estiveram associadas ao uso do Continue Positive Airway Pressure (CPAP), causando lesões no septo nasal, e em dois neonatos, ao posicionamento deles em sua acomodação, ocasionando lesões na região occipital. É fundamental que os enfermeiros identifiquem os riscos para o surgimento de úlceras por pressão e elaborem e/ou utilizem escalas para predição de risco, visando à melhora da qualidade da assistência e reduzindo as complicações decorrentes deste tipo de lesão, o tempo de hospitalização, a mortalidade e os custos para o tratamento.
\end{abstract}

DESCRITORES: Úlcera por pressão. Recém-nascido. Unidades de Terapia Intensiva Neonatal. Estomaterapia.

\begin{abstract}
The aim of this study was to describe the occurrence of pressure ulcers in newborns admitted to the Neonatal Intensive Care Unit of a public hospital in Brasília, in the Federal District, Brazil. This was an exploratory, descriptive and case series study, which was conducted between September and December 2014. Six newborns who developed pressure ulcer during their hospitalization period in the Neonatal Intensive Care Unit were evaluated. In four of the newborns, the ulcers were associated with the use of the Continue Positive Airway Pressure (CPAP), causing injuries in the nasal septum, and in two of them, they were associated with their position in bed, resulting in injuries in the occipital region. It is essential that the nurses identify the risks of development of pressure ulcers and elaborate and/ or use risk assessment scales for prediction, aiming to improve the care quality and decrease the complications from this kind of injury, hospitalization time, mortality, and treatment costs.
\end{abstract}

DESCRIPTORS: Pressure ulcer. Infant, newborn. Intensive Care Units, Neonatal. Stomatherapy.

\footnotetext{
*Extraído do Trabalho de Conclusão de Curso de Graduação intitulado "Incidência de lesões de pele em neonatos internados na Unidade de Terapia Intensiva Neonatal", apresentado ao Departamento de Enfermagem, Universidade de Brasília, em 2015.

${ }^{1}$ Mestranda do Programa de Pós-graduação em Enfermagem do Departamento de Enfermagem da Universidade de Brasília (UnB) - Brasília (DF), Brasil. Endereço para correspondência: Departamento de Enfermagem, Faculdade de Ciências da Saúde, Universidade de Brasília, Campus Universitário Darcy Ribeiro - CEP: 70910-900 - Asa Norte - Brasília (DF), Brasil- E-mail: talitafaraj@gmail.com

${ }^{2}$ Doutora em Enfermagem. Professora Adjunta do Departamento de Enfermagem da UnB - Brasília (DF), Brasil.

Artigo recebido em: 26/10/2015 - Aceito para publicação em: 23/12/2015
} 


\section{RESUMEN}

El objetivo de este estudio fue describir la ocurrencia de úlceras por presión en recién nacidos internados en una Unidad de Cuidado Intensivo Neonatal de un hospital público de Brasília, Distrito Federal, Brasil. Se trata de un estudio exploratorio de carácter descriptivo y serie de casos, realizado en el periodo de septiembre hasta diciembre del 2014. Se evaluaron seis recién nacidos que desarrollaron úlcera por presión durante su periodo de internación en la Unidad de Cuidado Intensivo Neonatal. En cuatro neonatos, las úlceras estuvieron asociadas al uso del Continue Positive Airway Pressure (CPAP), lo que causó lesiones en su septo nasal, y en dos neonatos las úlceras estuvieron asociadas al posicionamiento del recién nacido en su acomodación, resultando en lesiones en la región occipital. Es fundamental que los enfermeros identifiquen los riesgos para el surgimiento de úlceras por presión y elaboren y/o utilicen escalas de predicción de riesgo, con el intuito de la mejora de la calidad de la asistencia y para reducir las complicaciones decurrentes de este tipo de lesión, el tiempo de hospitalización, la mortalidad y los costos para el tratamiento.

DESCRIPTORES: Úlcera por presión. Recién nacido. Unidades de Cuidado Intensivo Neonatal. Estomaterapia.

\section{INTRODUÇÃO}

A pele compõe 13\% da superfície corporal do recém-nascido e sua fragilidade representa uma maior absorção transepidérmica de substâncias, um aumento das necessidades hídricas, o risco para instabilidade térmica, assim como maior colonização de microrganismos e infecção invasiva ${ }^{1}$. Aproximadamente $80 \%$ da morbidade e mortalidade dos neonatos está relacionada a traumas ou alterações da função normal da pele, como consequência de sua imaturidade funcional associada ao manejo inadequado dos profissionais que prestam a assistência ${ }^{1}$.

De acordo com estudos realizados em Unidades Neonatais, as lesões de pele mais frequentes nos recém-nascidos são: hematomas, eritema, escoriações, equimoses, pústulas, monilíase e infiltraçãa ${ }^{2,3}$. Em uma revisão de literatura ${ }^{4}$, a prevalência de úlcera por pressão (UP) na população pediátrica variou de 2 a $28 \%$, e a incidência de UP nesta população admitida na Unidade de Terapia Intensiva (UTI) foi de $26 \%$.

A UP é uma lesão localizada na pele, no tecido e/ou na estrutura subjacente, geralmente sobre proeminência óssea, que resulta de pressão isolada ou combinada com cisalhamento ${ }^{5}$. É considerada um agravo comum que dificulta a recuperação dos pacientes acometidos; prolonga o período de internação; aumenta o risco de infecções; gera custos elevados para os pacientes e para os serviços de saúde, além de contribuir para o aumento da taxa de mortalidade ${ }^{6}$.

A hospitalização de neonatos pode ser considerada um fator de risco para o surgimento das lesões de pele, incluindo as UP, em função de comprometimento da perfusão, imaturidade da pele, alteração do nível de consciência e da percepção sensorial, limitação da mobilidade espontânea, uso de dispositivos médicos, instabilidade hemodinâmica, retenção de líquidos, entre outros ${ }^{7}$.
É importante considerar a UP como uma complicação grave do paciente hospitalizado e, assim, a equipe multidisciplinar deve estar engajada na implantação de protocolos para este tipo de lesão, principalmente aos pacientes que estão mais expostos ao risco ${ }^{8}$. A manutenção da integridade da pele do neonato durante o período crítico é fundamental, uma vez que ela é uma barreira protetora dos órgãos internos e fatores como dermatites, úlceras, traumatismos, etc. podem prejudicar a função de proteção dessa membrana9.

\section{OBJETIVO}

O presente trabalho objetivou relatar a ocorrência de UP em recém-nascidos internados na UTI Neonatal de um hospital público de Brasília, no Distrito Federal, bem como descrever as suas características e os fatores associados ao seu surgimento.

\section{MÉTODO}

Trata-se de um estudo exploratório de caráter descritivo, do tipo série de casos, realizado na UTI Neonatal do Hospital Materno Infantil de Brasília (HMIB), no Distrito Federal, de setembro a dezembro de 2014. Foram incluídos os recém-nascidos que tinham, no máximo, 28 dias de vida no momento da admissão e que permaneceram internados por um período mínimo de 24 horas. Foram excluídos da amostra os recém-nascidos que os representantes legais se recusaram a assinar o termo de consentimento livre e esclarecido ou retiraram o seu consentimento. 
A amostra de conveniência foi constituída por seis recémnascidos que desenvolveram UP durante o seu período de internação na UTI. Os dados sociodemográficos e clínicos dos neonatos foram levantados por meio de análise dos prontuários. As informações referentes às UP também foram coletadas pelo exame dos prontuários e pela avaliação clínica ${ }^{5}$, por meio da observação direta dos neonatos durante a realização dos procedimentos de rotina do setor pela equipe de Enfermagem. Não houve o registro da utilização de escalas de avaliação de risco para o desenvolvimento de UP pelos profissionais.

O projeto de pesquisa foi submetido à avaliação do Comitê de Ética em Pesquisa da Fundação de Ensino e Pesquisa em Ciências da Saúde (FEPECS), sendo aprovado em 08 de setembro de 2014 pelo parecer consubstanciado 781.809 .

\section{RESULTADOS}

Dentre os neonatos estudados, três eram do sexo masculino e três do feminino. A maioria era procedente do Distrito Federal e apenas um participante era de outro estado (Tabela 1).

Em relação ao motivo da internação dos recémnascidos na UTIN, três tinham o diagnóstico médico de Síndrome do Desconforto Respiratório ou Doença da Membrana Hialina (SDR/DMH) e três tinham outros diagnósticos, tais como gastrosquise, septicemia bacteriana e asfixia perinatal.

Quanto ao surgimento das UP, quatro neonatos apresentaram lesão na região nasal e dois na occipital, conforme evidencia a Tabela 2 .

Tabela 1. Distribuição dos recém-nascidos segundo suas características sociodemográficas e clínicas, Brasília, Distrito Federal, 2014.

\begin{tabular}{|c|c|c|c|c|c|c|}
\hline RN & Sexo & $\begin{array}{l}\text { Peso ao } \\
\text { nascer }\end{array}$ & Procedência & $\begin{array}{l}\text { Tipo de } \\
\text { parto }\end{array}$ & $\begin{array}{l}\text { Total de dias de } \\
\text { internação }\end{array}$ & $\begin{array}{c}\text { Acomodação do RN } \\
\text { na UTI Neonatal }\end{array}$ \\
\hline 1 & M & $1.096 \mathrm{~g}$ & Palmital, Minas Gerais & Cesárea & 17 & Incubadora \\
\hline 2 & $F$ & $1.036 \mathrm{~g}$ & Riacho Fundo, Distrito Federal & Normal & 35 & Incubadora \\
\hline 3 & M & $1.665 \mathrm{~g}$ & Ceilândia, Distrito Federal & Normal & 38 & Incubadora \\
\hline 4 & M & $1.035 \mathrm{~g}$ & Riacho Fundo, Distrito Federal & Normal & Superior a 60 & Incubadora \\
\hline 5 & $F$ & $3.000 \mathrm{~g}$ & Planaltina, Distrito Federal & Cesárea & 18 & Berço aquecido \\
\hline 6 & $\mathrm{~F}$ & $3.645 \mathrm{~g}$ & Brazlândia, Distrito Federal & Cesárea & 19 & Berço aquecido \\
\hline
\end{tabular}

RN: recém-nascidos; M: masculino; F: feminino; UTI: Unidade de Terapia Intensiva.

Tabela 2. Distribuição dos recém-nascidos de acordo com as características das úlceras por pressão, Brasília, Distrito Federal, 2014.

\begin{tabular}{|c|c|c|c|c|}
\hline RN & $\begin{array}{l}\text { Dias de nascido } \\
\text { quando surgiu a UP }\end{array}$ & $\begin{array}{l}\text { Localização } \\
\text { da lesão }\end{array}$ & $\begin{array}{l}\text { Estadiamento } \\
\text { da UP }\end{array}$ & $\begin{array}{l}\text { Fatores de risco para o } \\
\text { surgimento da UP }\end{array}$ \\
\hline 1 & 9 & Nasal & Categoria II & $\begin{array}{l}\text { Uso do CPAP com pronga } \\
\text { nasal por mais de } 2 \text { dias }\end{array}$ \\
\hline 2 & 4 & Nasal & Categoria II & $\begin{array}{l}\text { Uso do CPAP com pronga } \\
\text { nasal por mais de } 2 \text { dias }\end{array}$ \\
\hline 3 & 10 & Nasal & Categoria II & $\begin{array}{l}\text { Uso do CPAP com pronga } \\
\text { nasal por mais de } 2 \text { dias }\end{array}$ \\
\hline 4 & 6 & Nasal & Categoria II & $\begin{array}{l}\text { Uso do CPAP com pronga } \\
\text { nasal por mais de } 2 \text { dias }\end{array}$ \\
\hline 5 & 12 & Occipital & Categoria II & $\begin{array}{l}\text { Ventilação mecânica; } \\
\text { mobilidade prejudicada }\end{array}$ \\
\hline 6 & 19 & Occipital & Categoria I & $\begin{array}{l}\text { Ventilação mecânica; } \\
\text { mobilidade prejudicada }\end{array}$ \\
\hline
\end{tabular}

RN: recém-nascidos; M: masculino; F: feminino; UTI: Unidade de Terapia Intensiva; UP: úlcera por pressão; CPAP: Continue Positive Airway Pressure. 
Os quatro recém-nascidos que desenvolveram UP na região nasal tiveram suas lesões associadas ao uso do Continue Positive Airway Pressure (CPAP), com erosão no septo e sangramento. Os neonatos que desenvolveram UP na região occipital tiveram suas lesões associadas ao posicionamento em sua acomodação, sendo que um deles apresentou eritema não branqueável na região acometida e o outro, perda parcial da espessura da pele, tendo suas lesões classificadas respectivamente como categorias I e II.

Em relação ao tratamento implementado para o tratamento das lesões, registrou-se uso do hidrocoloide extrafino apenas nas úlceras localizadas na região occipital.

\section{DISCUSSÃO}

Os danos causados por dispositivos como cateteres, tubos de oxigênio, ventilação mecânica, entre outros, podem ocasionar úlceras em locais pouco habituais no recém-nascido, como orelha, nariz e abdome ${ }^{10}$. Em um estudo realizado na UTIN de um Hospital Universitário em Pernambuco ${ }^{11}$, a incidência de lesão no septo nasal decorrente do uso de CPAP foi de $62,9 \%$ e, entre os recém-nascidos que tiveram lesão categoria II, foi de $36,4 \%$. Verificou-se que quanto maior o tempo de uso do CPAP, maior é a incidência das lesões de categorias II e III. Este é um fator preocupante já que os danos observados nesses estágios podem variar de necrose à perda total do septo nasal ${ }^{11}$.

Outro estudo realizado em uma Unidade Neonatal de Maceió $^{12}$ observou que todos os neonatos que utilizaram CPAP com pronga nasal por um período mínimo de dois dias tiveram lesões, sendo que, em 19,7\% destes recémnascidos, elas foram classificadas como moderadas, ou seja, apresentaram sangramento ou sangramento e erosão. No presente estudo, os quatro neonatos que desenvolveram úlcera pelo uso do CPAP eram prematuros e também apresentaram sangramento e erosão na região nasal, corroborando os dados citados.

Os recém-nascidos do presente trabalho que desenvolveram UP na região occipital estavam acomodados em berço aquecido. Um deles teve sua lesão classificada como categoria I e o outro como categoria II, coincidindo com dados de outro estudo ${ }^{13}$. Para prevenção das UP, recomenda-se o reposicionamento no leito a cada duas a três horas, conforme sua estabilidade, assim como o uso de colchões especiais, a inspeção frequente da pele, o manuseio adequado da pele do neonato, o uso de protetores sob os dispositivos, entre outros ${ }^{5,10,14}$.

É fundamental que os enfermeiros identifiquem os riscos para o surgimento de UP e elaborem e/ou utilizem escalas para predição de risco, visando à melhora da qualidade da assistência e reduzindo as complicações decorrentes deste tipo de lesão, o tempo de hospitalização, a mortalidade e os custos para o tratamento.

\section{CONCLUSÃO}

Os resultados deste relato evidenciam a necessidade da criação de protocolos e/ou a utilização de instrumentos que visem à manutenção da integridade da pele do recémnascido, avaliando-a criteriosa e continuamente. Estes podem ser ferramentas úteis para identificar qualquer alteração no sistema tegumentar do neonato e, assim, prevenir a ocorrência de UP e suas consequências.

Observou-se um número reduzido de estudos nacionais sobre a incidência e prevalência de UP em neonatos internados nas UTI Neonatais. Desse modo, espera-se que os dados apresentados por meio deste relato possam despertar o interesse de enfermeiros para o desenvolvimento de novos estudos incluindo recém-nascidos que estão nas condições semelhantes às que foram descritas.

\section{REFERÊNCIAS}

1. Martins CP, Tapia CEV. A pele do recém-nascido prematuro sob a avaliação do enfermeiro: cuidado norteando a manutenção da integridade cutânea. Rev Bras Enferm. 2009;62(5):778-83.

2. Nepomuceno LMR, Kurcgant P. Uso de indicador de qualidade para fundamentar programa de capacitação de profissionais de enfermagem. Rev Esc Enferm USP. 2008;42(4):665-72.
3. Fontenele FC, Cardoso MV. Lesões de pele em recémnascidos no ambiente hospitalar: tipo, tamanho e área afetada. Rev Esc Enferm USP. 2011;45(1):130-7.

4. Kottner J, Wilborn D, Dassen T. Frequency of pressure ulcer in the paediatric population: a literature review and new empirical data. Int J Nursing Studies. 2010;47(10):1330-40. 
5. Haesler E (ed.). European Pressure Ulcer Advisory Panel. National Pressure Ulcer Advisory Panel. Pan Pacific Pressure Injury Alliance. Prevention and treatment of pressure ulcers: quick reference guide. Australia: Cambridge Media; 2014.

6. Sales MC, Borges EL, Donoso MT. Risco e prevalência de úlceras por pressão em uma unidade de internação de um hospital universitário de Belo Horizonte. Rev Min Enferm. 2010;14(4):566-75.

7. Visscher M, Taylor T. Pressure ulcer in the hospitalized neonate: rates and risk factors. Sci Reports. 2014;4:7429.

8. Medeiros $A B$, Lopes $C H$, Jorge MS. Análise da prevenção e tratamento das úlceras por pressão propostos por enfermeiros. Rev Esc Enferm USP. 2009;43(1):223-8.

9. Fontenele FC, Pagliuca LM, Cardoso MV. Cuidados com a pele do recém-nascido: análise de conceito. Esc Anna Nery. 2012;16(3):480-5.
20. Santos SV, Costa R. Prevenção de lesões de pele em recém-nascidos: o conhecimento da equipe de enfermagem. Texto Contexto Enferm. 2015;24(3):731-9.

21. Bomfim SF, Vasconcelos MG, Sousa NF, Silva DV, Leal LP. Lesão de septo nasal em neonatos pré-termo no uso de prongas nasais. Rev Latino-Am Enferm. 2014;22(5):826-33.

22. Nascimento RM, Ferreira $A L$, Coutinho AC, Veríssimo RC. Frequência de lesão nasal em neonatos por uso de pressão positiva contínua nas vias aéreas com pronga. Rev Latino-Am Enferm. 2009;17(4):489-94.

23. Crozeta K, Stocco JGD, Danski MT, Meier MJ. Úlceras por pressão em neonatos e crianças: perfil epidemiológico e clínico. Rev Min Enferm. 2010;14(2):233-8.

24. Ness MJ, Davis DM, Carey WA. Neonatal skin care: a concise review. Int J Dermatol. 2013;52(1):14-22. 